УДК 620.179 .119

\title{
Investigation Natural Vibrations Rail Lashes \\ for Organization of Emergency Acoustic Channel \\ Communications in the Mines \\ and Detection of Defects
}

\author{
Vadim S. Potylitsyn, Danil S. Kudinov, \\ Konstantin A. Artemyev and Ekaterina A. Kokhonkova* \\ Siberian Federal University \\ 79 Svobodny, Krasnoyarsk, 660041, Russia
}

Received 12.06.2016, received in revised form 18.08.2016, accepted 06.11.2016

This article discusses aspects of application parameters natural frequency objects. The calculated and experimental results to determine the natural frequencies of the rails and the estimation of damping vibrations at these frequencies. We investigate radar $(R L)$ method is to obtain information about the defects of the rail track by registering their own frequency mechanical vibrations between crosstie rail span.

Keywords: defectoscopy of rails, mine emergency communication, the natural frequency, elastic vibration, attenuation.

Citation: Potylitsyn V.S., Kudinov D.S., Artemyev K.A., Kokhonkova E.A. Investigation natural vibrations rail lashes for organization of emergency acoustic channel communications in the mines and detection of defects, J. Sib. Fed. Univ. Eng. technol., 2016, 9(7), 1131-1138. DOI: 10.17516/1999-494X-2016-9-7-1131-1138.

(C) Siberian Federal University. All rights reserved

* Corresponding author E-mail address: kudinovdanil@yandex.ru 


\title{
Исследование собственных колебаний рельсовых плетей
}

\section{для организации аварийного}

акустического канала связи

в шахтах и обнаружения дефектов

\author{
В.С. Потылицын, Д.С. Кудинов, \\ К.А. Артемьев, Е.А. Кохонькова \\ Сибирский федеральный университет \\ Россия, 660041, Красноярск, пр. Свободный, 79
}

Рассматриваются прикладные аспекты применения параметров частоты собственных механических (ЧСК) объектов. Приведень расчетные и экспериментальные результатьл по определению частот собственных колебаний рельсов и оценка затухания колебаний на этих частотах. Исследуется радиолокационный (РЛ) метод, заключаюшийся в получении информации о дефектах рельсового фрагмента путём регистрации колебаний ЧСК междушпального пролёта рельса.

Ключевые слова: дефектоскопия рельсов, аварийная шахтная связь, частота собственных колебаний, упругие колебания, затухание.

\section{Введение}

Роль неразрушающего контроля (НК) на современных железных дорогах чрезвычайно актуальна. Основными средствами дефектоскопии на железнодорожном транспорте являются электромагнитные и акустические дефектоскопы. Преимущество первых - возможность ведения бесконтактного контроля при движении на скорости до 80 км/ч, однако использование вихретокового принципа построения электромагнитных дефектоскопов не позволяет обнаруживать дефекты рельса при глубине их залегания более 8 мм. Достоинством акустических, или ультразвуковых (УЗ), дефектоскопов служит их высокая проникающая способность, что предопределяет их использование в качестве основных средств дефектоскопии рельсового пути. Однако УЗ-дефектоскопы требуют наличия физического контакта между пьезопреобразователем и контролируемым участком рельса $[1,2]$.

Предлагается подход, заключающийся в получении информации о дефектах рельсового фрагмента путём регистрации частоты собственных механических колебаний участка пути. ЧСК любого физического макрообъекта зависит от его механических характерстик, таких как модуль упругости, плотность материала и т.д., а также от геометрической формы, наличия неоднородностей и т. п. Очевидно, что для тех рельсовых фрагментов, параметры которых совпадают с эталонными, ЧСК будут идентичны. Наличие дефекта вносит изменение в структуру рельса, что отражается на спектре собственных колебаний.

Для обоснования бесконтактного метода диагностики, заключающегося в регистрации ЧСК рельсов, воспользуемся теорией колебаний упругих стержней. Для этого междушпальный участок рельса будем рассматривать в качестве жёсткого упругого стержня, закреплённого на двух неподвижных опорах. Выражение для ЧСК будет иметь вид: 
a
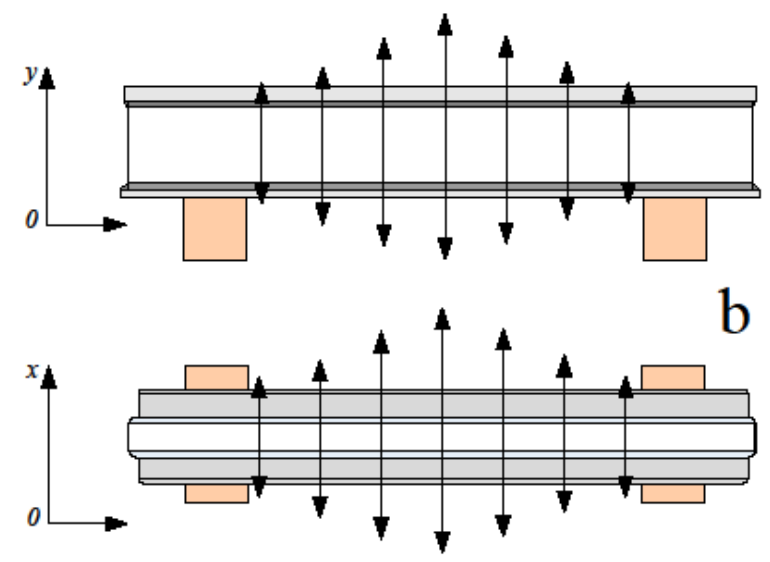

Рис. 1. Поперечные колебания рельса при ударном возбуждении: а - в вертикальной плоскости $y 0 z$; б - в горизонтальной плоскости $x 0 z$

$$
f=\frac{\pi}{2 \cdot l^{2}} \sqrt{\frac{E \cdot J}{S \cdot \rho}},
$$

где $l$ - длина стержня; Е - модуль упругости материала; $\mathrm{S}$ - площадь сечения рельса; J - момент инерции сечения [3].

Дадим теоретическую и экспериментальную оценку ЧСК участка рельса, жёстко закреплённого на двух неподвижных опорах. При этом необходимо учесть, что рельс совершает поперечные колебания в двух взаимно перпендикулярных плоскостях: вертикальной и горизонтальной (рис. 1).

Для анализа выбраны рельсы марки Р33, используемые в шахтных выработках, с параметрами:

- длина пролета рельса $l=0,5 \mathrm{M}$;

- площадь поперечного сечения рельса $S=0,427 \mathrm{M}^{2}$;

- момент инерции $J_{x}=967,9 \mathrm{~cm}^{4}, J_{y}=166,7 \mathrm{~cm}^{4}$;

- модуль Юнга $E=2,1 \cdot 10^{11}$ Па;

- плотность $\rho=7830 \mathrm{\kappa} / \mathrm{M}^{3}$ [4].

Таким образом, получим ЧСК в горизонтальной и вертикальной плоскостях соответственно:

$$
\begin{aligned}
& f_{Z O X}=\frac{\pi}{2 \cdot l^{2}} \sqrt{\frac{E \cdot J_{X O Z}}{S \cdot \rho}}=\frac{\pi}{2 \cdot 0.5^{2}} \sqrt{\frac{2.1 \cdot 10^{11} \cdot 9.7 \cdot 10^{-5}}{43 \cdot 10^{-4} \cdot 7830}}=7680 \Gamma u, \\
& f_{Y O X}=\frac{\pi}{2 \cdot l^{2}} \sqrt{\frac{E \cdot J_{X O Y}}{S \cdot \rho}}=\frac{\pi}{2 \cdot 0.5^{2}} \sqrt{\frac{2.1 \cdot 10^{11} \cdot 1.7 \cdot 10^{-5}}{43 \cdot 10^{-4} \cdot 7830}}=2045 \Gamma u .
\end{aligned}
$$

Две компоненты упругой волны распространяются в безграничном полупространстве по закону 


$$
\begin{gathered}
A_{Z}=\frac{A_{0}}{\sqrt{k_{R} x}} \cdot e^{-x x} \cdot\left(e^{-\frac{\omega k_{1} z}{V_{R}}} \cdot\left(\frac{V_{R}}{V_{S}}\right)^{2}-2+2 k_{1} k_{2} e^{-\frac{\omega k_{1} z}{V_{R}}}\right), \\
A_{Y}=\frac{A_{0}}{\sqrt{k_{R} x}} \cdot e^{-\gamma x} \cdot\left(e^{-\frac{\omega k_{1} z}{V_{R}}} \cdot\left(\frac{V_{R}}{V_{S}}\right)^{2}-2+2 e^{-\frac{\omega k_{1} z}{V_{R}}}\right),
\end{gathered}
$$

где $A_{Z}, A_{Y}$ - компоненты смещения для продольной и поперечной волны; $A_{0}$ - начальное смещение среды под действием источника колебаний; $\omega=f \cdot 2 \pi$ - круговая частота; $k_{R}-$ волновое число; $x-$ расстояние от источника до приемника; $\gamma=3,9 \cdot 10^{-4} \mathrm{M}^{-1}-$ коэффициент поглощения упругих волн в среде (зависит от частоты); $z$ - глубина измерения; $k_{1}=\sqrt{1-\left(V_{R} / V_{p}\right)^{2}}, k_{2}=\sqrt{1-\left(V_{R} / V_{S}\right)^{2}} ; V_{s}, V_{p}$, $V_{R}$ - скорость поперечной, продольной и поверхностной волн [5].

Так как волна распространяется в рельсе как в звуковом волноводе и измерения проводятся при $z=0$, затуханием, связанным с расхождением волнового пучка, можно пренебречь:

$$
\begin{gathered}
A_{Z}=A_{0} \cdot e^{-\gamma x} \cdot\left(\frac{V_{R}}{V_{S}}\right)^{2}-2+2 k_{1} k_{2}, \\
A_{Y}=A_{0} \cdot e^{-\gamma x} \cdot\left(\frac{V_{R}}{V_{S}}\right)^{2} .
\end{gathered}
$$

Для частот 2045 и 7680 Гц оценим затухание колебаний в рельсе. В акустическом датчике смещение среды в упругих волнах преобразовывается в напряжение через параметр чувствительности $G=27,5 \mathrm{~B} / \mathrm{M} / \mathrm{c}$ (3).

$$
\begin{aligned}
& U_{Z}=A_{Z} \cdot \omega \cdot G, \\
& U_{Y}=A_{Y} \cdot \omega \cdot G .
\end{aligned}
$$

Расчетные графики затухания сигнала для продольных и поперечных колебаний в рельсе в зависимости от расстояния на частотах 2045 и 7680 Гц представлены на рис. 2. Начальные значения смещения компонент $A_{Z}$ и $A_{Y}$ разные, а характер затухания одинаковый.

Приведенные расчеты и экспериментальные оценки показывают, что для передачи полезного сигнала на большое расстояние возможно использование рельсов, так как затухание на одной из ЧСК в них мало. Данный факт можно использовать для передачи данных в аварийных ситуациях в шахтах.

Сравнивая величины затухания колебаний вертикальной и горизонтальной составляющей (рис. 2), можно сделать вывод, что более целесообразно возбуждать сигнал на частоте колебаний горизонтальной составляющей $\left(f_{y o x}=2045\right.$ Ги) путем установки вибратора на шейку рельса, так как затухание для неё на 32 дБ меньше.

В работе [1] приведены расчетные и экспериментальные оценки ЧСК для рельса марки Р65 с длиной $l=1,7$ м. Из выражения (1) были получены частоты для вертикальной $-f_{\text {ҮOX }} \approx 1,7$ кГЦ и горизонтальной плоскости $-f_{Y O X} \approx 650$ ГЦ. Результаты эксперимента представлены в виде временной и спектральной характеристики процесса свободных затухающих колебаний рельса при воздействии одиночного импульса (рис. 3).

Для оценки влияния дефекта на ЧСК рельса целесообразно рассматривать изменение не только основной частоты $v_{0}$ в спектре колебаний, а смещение всех колебательных мод, при- 


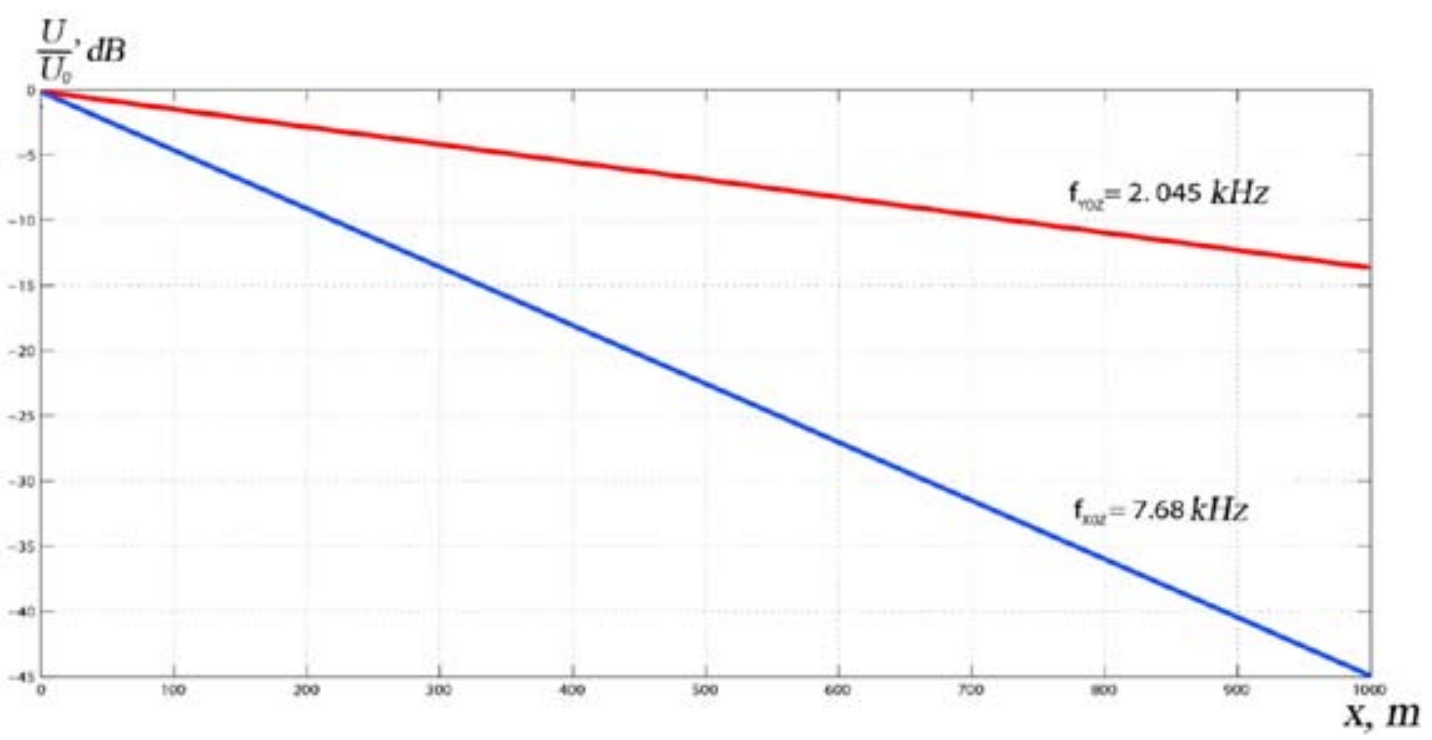

Рис. 2. Затухание колебаний с расстоянием в рельсе модели Р65 на резонансных частотах 2045 и 7680 Гц
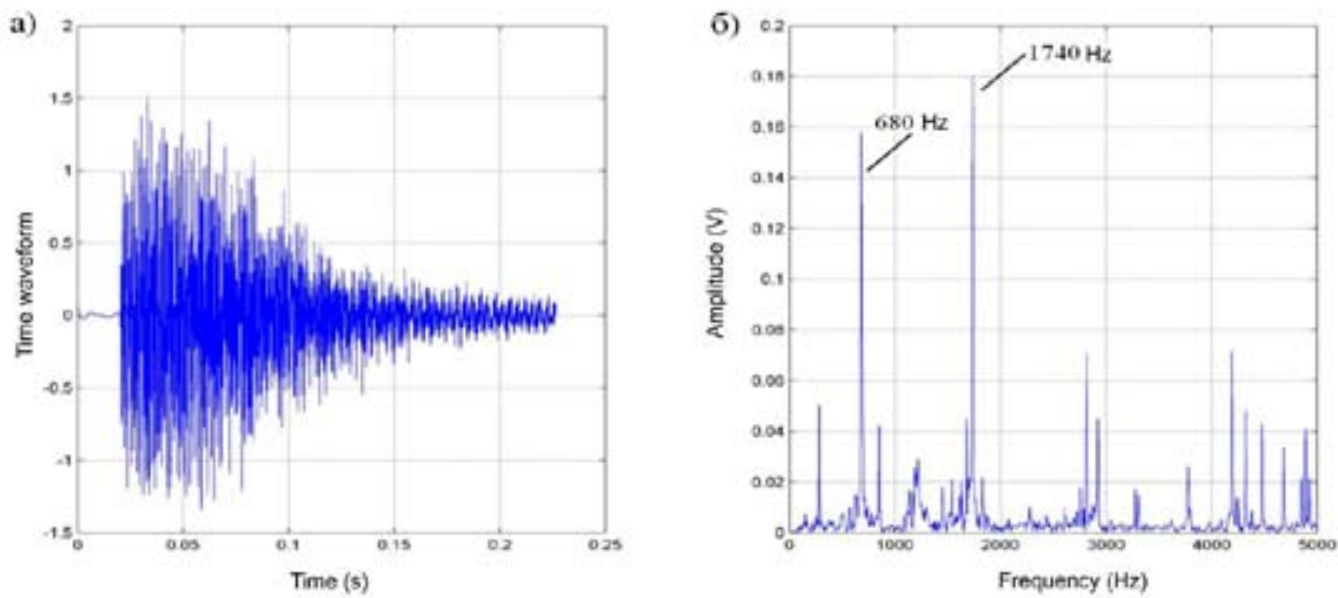

Рис. 3. Характеристики процесса свободных колебаний рельса: а - осциллограмма свободных колебаний рельса; б - спектр свободных колебаний рельса

сутствующих в спектре. Для выявления зависимости смещения колебательных мод в спектре ЧСК от наличия дефекта был проведен эксперимент по регистрации смещения ЧСК дефектного образца. Для этого в головке рельса искусственно создавался дефект в виде запила шириной

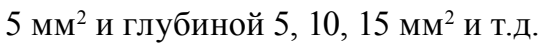

В результате было установлено, что увеличение дефекта в головке рельса на каждые 5 мм $^{2}$ отражается на изменении ЧСК рельса примерно на 0,05 \%, что соответствует смещению каждой колебательной моды спектра на 1-3 Гц по сравнению с предыдущим значением при увеличении размера дефекта на 5 мм². $^{2}$ 
Кроме того, было выявлено, что в спектре дефектного образца возникают дополнительные колебательные моды, которые также могут свидетельствовать о наличии структурной неоднородности.

В настоящее время методы НК, основанные на получении информации о дефектах путём анализа спектральных характеристик ЧСК объектов контроля, получают широкое распространение, в том числе на железнодорожном транспорте. В качестве датчиков для регистрации параметров ЧСК используются, как правило, микрофонные сборки (МС). Недостаток МС в том, что они обладают низкой направленностью и поэтому подвержены влиянию внешних акустических шумов. Это позволяет давать с их помощью лишь приближённую интегральную оценку технического состояния обследуемого узла, но не даёт возможности с достаточной степенью точности выявлять дефект конкретной детали. С использованием РЛ датчиков можно будет осуществлять быстрое направленное дистанционное сканирование, что даст возможность производить селективный контроль деталей и узлов ПС в движении на высокой скорости.

Аппаратная реализация бесконтактного дефектоскопа железнодорожных путей в движении, построенного на принципе выделения информации из СВЧ-радиоволн, модулированных параметрами собственных механических колебаний рельса, является весьма сложной задачей в силу влияния помех, связанных с движением дефектоскопа. Сам процесс радиолокационного считывания механических колебаний можно представить следующим образом.

В состоянии покоя отражённый сигнал на приёмной антенне будет иметь следующий вид:

$$
U(t)=U_{\Pi P} \sin \left(\omega t+\varphi_{0}\right),
$$

где $\omega$ - частота радиосигнала; $\varphi_{0}$ - начальная фаза радиосигнала, которая определяется соотношением

$$
\varphi_{0}=\frac{2 \pi \cdot r}{\lambda}
$$

где $r$ - расстояние от излучателя до объекта исследования; $\lambda$ - длина волны радиосигнала.

Если объект контроля совершает колебания с частотой $\Omega$, то в соответствии с периодическим изменением параметра $r$ в выражении (7) будет изменяться параметр фазы $\varphi_{0}$. Перепишем выражение (7) для данного случая:

$$
U(t)=U_{\Pi P} \sin \left(\omega t+\Delta \varphi+\varphi_{0}\right)
$$

где $\Delta \varphi=\frac{2 \pi \cdot r_{0} \sin (\Omega t)}{\lambda}-$ параметрическая зависимость изменения фазы радиосигнала от колебательного смещения поверхности рельса; $\Delta r=r_{0} \sin (\Omega t)$ - смещение облучаемой поверхности рельса; $\varphi_{0}$ - стационарная часть фазового смещения; $\Omega$ - частота вибраций рельса.

Тогда радиосигнал на приёмной антенне описывается выражением

$$
U(t)=U_{\Pi P} \sin \left(\omega t+\frac{2 \pi \cdot \Delta r(\Omega)}{\lambda}+\varphi_{0}\right)
$$

Рассмотрим вариант реализации системы дефектоскопии деталей и узлов подвижного состава на основе регистрации ЧСК радиолокационным методом (рис. 4a). В этом случае при движении поезда происходит радиолокационное сканирование узлов ПС (колёсные пары, бук- 


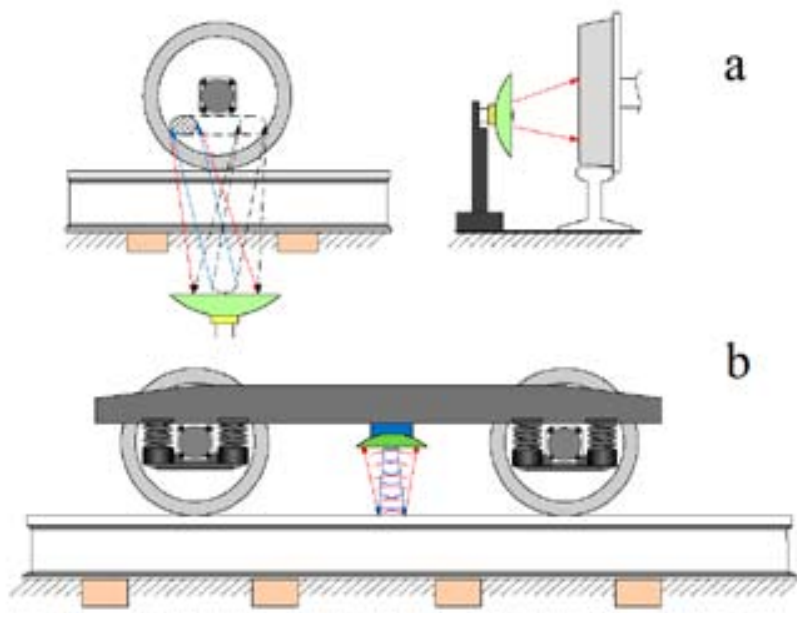

Рис. 4. РЛ-метод бесконтактной дефектоскопии: а - узлов подвижного состава со стационарной установкой; б - рельсов с установкой на движущемся подвижном составе

совые узлы, автосцепки и т.д.) и выделение спектра ЧСК, который сравнивается с эталонным спектром бездефектного образца, на основе чего принимается решение о дефектности обследуемого узла.

На рис. $4 б$ изображена структурная схема работы РЛ бесконтактного дефектоскопа для неразрушающего контроля (НК) рельсового пути в движении. При движении поезда возникает акустический шум, в спектре которого будут присутствовать ЧСК рельса. Во время движения вибрирующая поверхность рельса облучается радиолокационным сигналом СВЧ-диапазона. Отражённый от вибрирующей поверхности радиосигнал будет содержать в себе информацию о параметрах колебаний рельса.

Чтобы обеспечить режим работы приёмопередающей антенны за пределами ближней зоны излучения при рабочей длине волны радиолокатора $\lambda=3 \mathrm{~cm}$ (рабочая частота $f=10 \Gamma \Gamma u$ ), РЛ устанавливается на мобильном средстве дефектоскопии на высоте около 0,5 м от облучаемой поверхности рельса.

\section{Заключение}

1. Приведённые теоретические и экспериментальные оценки показывают, что возможна реализация бесконтактного средства дефектоскопии ж/д путей, в основе которого лежит принцип сканирования рельса регистрацией параметрической модуляции отражённых от вибрирующей поверхности СВЧ радиоволн при помощи РЛ-датчика.

2. Для определения потенциальных возможностей метода необходимо провести измерение шумов движения в натурных условиях, а именно оценить уровень соотношения сигнал/ помеха при установке опытного образца дефектоскопа на движущемся составе, а также определить частотный спектр шумов, связанных с движением.

3. По приведённым теоретическим оценкам можно заключить, что при возбуждении упругих колебаний на ЧСК возможна реализация аварийного канала связи, так как затухание упругих колебаний на частоте 2045 Гц составляет не более 13 дБ / 1 км. 
4. Кроме того, существующую упрощенную модель ЧСК рельса требуется дополнить с учетом влияния демпфирующих свойств подстилающей поверхности.

Проект поддержан за счет средств, предоставленных РФФИ, Правительством Красноярского края, Краевым фондом науки.

\section{Список литературы}

[1] Кудинов Д.С., Шайдуров Г.Я. Проблемы неразрушающего контроля рельсовых путей на железнодорожном транспорте. Датчики и Cистемь, 2009, 1(10), 19-27 [Kudinov D. S., Shaidurov G. Y., Non-destructive testing of track problems on the railways. Sensors and Systems. 2009, 1 (10), 19-27 (in Russian)]

[2] Еременко В. Т., Тютякин А. В. Методологические аспекты профилей сбора и обработки данных в системах неразрушающего контроля и диагностики технических объектов. Контроль. Диагностика, 2013, 1(1), 24-31 [Eremenko V. T., Tyutyakin A. V., Methodological aspects of data collection and processing profiles in the systems of nondestructive testing and diagnostics of technical objects. Control. Diagnostics. 2013, 1 (1), 24-31 (in Russian)]

[3] Лэмб Г. Динамическая теория звука. Л.: Наука, 1960. 372 [Lamb G., Dynamic sound theory. L .: Nauka, 1960, 372 (in Russian)]

[4] Бучнев В.К. Справочник горного инженера. Л.: Государственное научно-техническое издательство по горному делу, 1960. 791 [V. K. Buchnev, Reference book of a mining engineer. L .: State scientific and technical publishing house of mining, 1960, 791 (in Russian)]

[5] Викторов А. И. Поверхностные волны в твердых телах. Л.: Наука, 1981. 288 [Viktorov A. I., Surface waves in solids. L .: Nauka,, 1981, 288 (in Russian)]

[6] Гринёв А.Ю., Андриянов А.В., Багно Д.В. Многоканальный сверхширокополосный короткоимпульсный радар подповерхностного зондирования. Усnехи современной радиоэлектроники, 2009, 1(2), 19-27 [Grinyov A. Y., Andrianov A. V., Bagno D. V., Multi-channel ultrawideband short-pulse radar subsurface flexing. The success of modern electronics, 2009, 1 (2), 19-27 (in Russian)]

[7] Помозов В.В. Антенная система георадарного комплекса для мониторинга балластной призмы железнодорожного пути. Успехи современной радиоэлектроники, 2009, 2(1), 162-166 [Pomozov V. V., GPR antenna system for monitoring complex prism ballast railway track. The success of modern electronics. 2009, 2 (1), 162-166 (in Russian)]

[8] Гарсия Г., Дэвис Д., Методы неразрушающего контроля состояния рельсов. Железные дороги мира, 2003, 1(9), 18-21 [G. García, D. Davis, Methods of non-destructive testing of rails condition. The railways in the world. 2003, 1 (9), 18-21 (in Russian)]

[9] Вериго М.Ф., Коган А.Я. Взаимодействие пути и подвижного состава. Л.: Транспорт, 1997. 326 [Verigo M. F., Cohen A. J., Interaction track and rolling stock. L .: Transport, 1997. 326 (in Russian)]

[10] Сколник. М.И. Справочник по радиолокации. Т 2. Л.: Техносфера, 2014. 672 [Skolnick M. I., Reference book of radar: 2 volume. L .: Tehnocfera, 2014. 672 (in Russian)] 